\title{
Effectiveness of interventions to reduce household air pollution from solid biomass fuels and improve maternal and child health outcomes in low- and middle- income countries: a systematic review protocol
}

Katherine E. Woolley ${ }^{1+} \mathbb{D}$, Emma Dickinson-Craig ${ }^{1+}$, Suzanne E. Bartington ${ }^{1 *}$, Tosin Oludotun ${ }^{1}$, Bruce Kirenga ${ }^{2}$, Shelton T. Mariga ${ }^{2}$, Telesphore Kabera ${ }^{3}$, April Coombe ${ }^{1}$, Francis D. Pope ${ }^{4}$, Ajit Singh ${ }^{4}$, William R. Avis ${ }^{5}$, Rosie Day ${ }^{4}$, David Warburton ${ }^{6}$, Semira Manaseki-Holland ${ }^{1}$, David J. Moore' and G. Neil Thomas'

\begin{abstract}
Background: A variety of public health interventions have been undertaken in low- and middle-income countries (LMICs) to prevent morbidity and mortality associated with household air pollution (HAP) due to cooking, heating and lighting with solid biomass fuels. Pregnant women and children under five are particularly vulnerable to the effects of HAP, due to biological susceptibility and typically higher exposure levels. However, the relative health benefits of interventions to reduce HAP exposure among these groups remain unclear. This systematic review aims to assess, among pregnant women, infants and children (under 5 years) in LMIC settings, the effectiveness of interventions which aim to reduce household air pollutant emissions due to household solid biomass fuel combustion, compared to usual cooking practices, in terms of health outcomes associated with HAP exposure.

Methods: This protocol follows standard systematic review processes and abides by the PRISMA-P reporting guidelines. Searches will be undertaken in MEDLINE, EMBASE, CENTRAL, WHO International Clinical Trials Registry Platform (ICTRP), The Global Index Medicus (GIM), ClinicalTrials.gov and Greenfile, combining terms for pregnant women and children with interventions or policy approaches to reduce HAP from biomass fuels or HAP terms and LMIC countries. Included studies will be those reporting (i) pregnant women and children under 5 years; (ii) fuel transition, structural, educational or policy interventions; and (iii) health events associated with HAP exposure which occur among pregnant women or among children within the perinatal period, infancy and up to 5 years of age. A narrative synthesis will be undertaken for each population-intervention-outcome triad stratified by study design. (Continued on next page)
\end{abstract}

\footnotetext{
* Correspondence: S.Bartington@bham.ac.uk

${ }^{\dagger}$ Katherine E. Woolley and Emma Dickinson-Craig are joint first authors.

'Institute of Applied Heath Research, University of Birmingham, Birmingham,

UK

Full list of author information is available at the end of the article
}

(c) The Author(s). 2021 Open Access This article is licensed under a Creative Commons Attribution 4.0 International License, which permits use, sharing, adaptation, distribution and reproduction in any medium or format, as long as you give appropriate credit to the original author(s) and the source, provide a link to the Creative Commons licence, and indicate if changes were made. The images or other third party material in this article are included in the article's Creative Commons licence, unless indicated otherwise in a credit line to the material. If material is not included in the article's Creative Commons licence and your intended use is not permitted by statutory regulation or exceeds the permitted use, you will need to obtain permission directly from the copyright holder. To view a copy of this licence, visit http://creativecommons.org/licenses/by/4.0/ The Creative Commons Public Domain Dedication waiver (http://creativecommons.org/publicdomain/zero/1.0/) applies to the data made available in this article, unless otherwise stated in a credit line to the data. 


\begin{abstract}
(Continued from previous page)
Clinical and methodological homogeneity within each triad will be used to determine the feasibility for undertaking meta-analyses to give a summary estimate of the effect for each outcome.

Discussion: This systematic review will identify the effectiveness of existing HAP intervention measures in LMIC contexts, with discussion on the context of implementation and adoption, and summarise current literature of relevance to maternal and child health. This assessment reflects the need for HAP interventions which achieve measurable health benefits, which would need to be supported by policies that are socially and economically acceptable in LMIC settings worldwide.
\end{abstract}

Systematic review registration: PROSPERO CRD42020164998

Keywords: Indoor air pollution, Interventions, Low- and middle-income countries, Pregnancy outcomes, Child health, Maternal health, Environmental health, Biomass, Infant health

\section{Background}

Household cooking, heating and lighting with solid biomass fuel (e.g. wood, dung, charcoal, crop residues) [1] is common in low- and middle-income countries (LMICs) [2] worldwide, producing hazardous levels of household air pollution (HAP) (e.g. carbon monoxide (CO) and particulate mMatter (PM)) [3], and exposure to results in significant morbidity and mortality. The greatest burden of HAP exposure is recognised to be among women of child-bearing age [4] and children under 5 years, due to a disproportionate amount of time spent in the house, with women performing or assisting with household duties [5]. Intrauterine, infancy and early childhood are critical periods of organ development when individuals are particularly vulnerable to the harms of HAP exposure [6]. Adverse health events associated with HAP exposure can occur throughout the life course from conception to old age, but specifically during pregnancy, with evidence for increased risk of low birth weight, preterm birth, stillbirth, gestational hypertension, intrauterine growth retardation (IUGR) and perinatal mortality [7]. Early life health events among infants and children under 5 years include increased risks of acute lower respiratory infection (ALRI), asthma, otitis media, impaired neurodevelopment and all-cause mortality $[8,9]$. In context, 31.75 per 100,000 acute respiratory infection (ARI) deaths and 11.68 per 100,00 preterm birth deaths were attributable to HAP globally in 2019 [10].

Ultimately, economic development is associated with clean fuel transitions (e.g. to ethanol, liquid petroleum gas (LPG), electricity), which are fuels that have been recognised to reduce HAP levels to below World Health Organization indoor air quality (WHO-IAQ) guideline levels $\left(\mathrm{CO} 7 \mathrm{mg} / \mathrm{m}^{3}\right.$ 24-h average, $\mathrm{PM}_{2.5} 25 \mathrm{mg} / \mathrm{m}^{3}$ annual average) [11]; however, socio-cultural factors can contribute to fuel/stove stacking and mixing (where traditional fuels/stoves are used alongside the intervention) which may reduce the efficacy of clean fuel policy implementation [12]. Interim interventions, prior to sustained cleaner fuel availability, to mitigate HAP exposure levels within the household setting are broad ranging, including improved cookstoves (ICS) (e.g. rocket stoves, plancha) [13], solar stoves [14], improved biomass fuels (e.g. briquettes, biomass pellets) [13] and behavioural changes (e.g. removal of the child from the cooking area, outdoor cooking, opening windows) [12]. LPG, for example, has the potential to reduce HAP levels below the WHO-IAQ guideline levels; however, not all interventions achieve this [15-17] or interim targets $\left(\mathrm{PM}_{2.5} 35 \mathrm{mg} / \mathrm{m}^{3}\right.$ annual average) [18] and are therefore typically harm mitigation measures, with some interventions not reducing exposure at all. In addition, there are often multiple barriers [19] to implementation, uptake and sustained use of interventions, such as fuel affordability and accessibility, cultural and social preferences or lack of relevant infrastructure [20]. Previous systematic reviews have detailed the change in HAP levels [13] and health outcomes (low birth weight, preterm birth, perinatal mortality, paediatric ALRI and chronic obstructive pulmonary disease (COPD)) attributed to ICS interventions [21], in addition to systematic reviews investigating a wider range of HAP interventions for specified symptoms (e.g. blood pressure) [22] and general respiratory and nonrespiratory health outcomes [17]. However, to the best of our knowledge, there is a paucity of evidence synthesis concerning the overall benefits to maternal and child health outcomes arising as a consequence of household solid biomass fuel interventions.

The objective of the systematic review protocol outlined here is to assess, among pregnant women, infants and children (under 5 years) in LMIC settings, the effectiveness of interventions which aim to reduce household air pollutant emissions due to household solid biomass fuel combustion, compared to usual cooking practices, in terms of health outcomes associated with HAP exposure. In addition, any information regarding measures of sustained uptake of the intervention within the selected studies will be extracted and discussed. The findings will inform future intervention studies and policy changes, by generating knowledge of effectiveness for 
achieving improved pregnancy, perinatal, infant and under 5 years child health outcomes in resource-poor settings worldwide.

\section{Methods}

Established systematic review methods will be used. This protocol has been registered on the International Prospective Register of Systematic Reviews (PROSPERO) (ID: CRD42020164998) [23] and is presented in accordance with Preferred Reporting Items for Systematic Reviews and Meta-Analyses Protocol (PRISMA-P) guidelines [24].

\section{Eligibility criteria}

The following Population-Intervention-Comparator-Outcome-Study design (PICOS) criteria will be used to determining primary study inclusion.

\section{Population}

Pregnant women (no limitation to trimester or number of previous pregnancies), children in infancy and children under the age of 5 years who are exposed to HAP originating from biomass solid fuel sources, used for cooking, heating and lighting within LMIC settings (World Bank definition 2020) [25]. HAP exposure can be determined through direct objective measurement (e.g. personal, kitchen area) of pollutant concentration (e.g. PM, CO) or use of a proxy measure (e.g. selfreported biomass fuel use, classification of 'cleaner' and 'dirty' fuels by household survey).

\section{Intervention}

Any intervention implemented which aims to reduce household air pollution emissions arising from indoor cooking or heating using solid biomass fuel. This includes interventions such as those which seek to improve access and take-up to cleaner fuels (e.g. refined biomass, ethanol, LPG, solar, electricity); structural interventions such as improved cookstoves (ICS), inbuilt stoves (e.g. plancha), ventilation and chimney hood; fuel policy; and behavioural/ educational interventions (e.g. moving cooking outside, reducing time spent in the kitchen, removing children from the cooking area during cooking, altering fuel or food preparation). There will be no limitation to the length of duration of interventions or timing of deployment of intervention (e.g. anytime during pregnancy through to the fifth year of a child's life).

\section{Comparator}

Alternative HAP intervention (e.g. any other intervention within inclusion criteria) or no intervention (e.g. exposure to standard HAP through using the current method of cooking, heating or lighting).

\section{Outcomes}

Health outcomes relating to pregnancy and perinatal period (e.g. IUGR, birth weight, preterm birth, preeclampsia, pregnancy-induced hypertension, maternal mortality, perinatal/infant mortality, stillbirth and miscarriage) and early life (e.g. upper and lower respiratory tract infections, pneumonia, asthma, respiratory distress syndrome, otitis media, impaired neurodevelopment, mortality and burns) which have been previously associated with HAP exposure. There will be no limits to the follow-up duration of outcome measures.

\section{Study type}

Eligible study designs are randomised control trials (RCTs), non-randomised control trials and quasiexperimental or natural experimental studies (before-after studies, interrupted time-series studies). Time-series or before-and-after studies will need to compare the same health outcomes in the same population pre- and postintervention. It is recognised that before-and-after studies assessing pregnancy outcomes are unlikely to exist due to the difficulties in assessing changes in pregnancy outcomes within subsequent pregnancies, but will not be excluded if present.

\section{Exclusion}

Any study that did not meet the inclusion criteria in all five areas (population, intervention, comparator, outcomes and study design) will be excluded.

\section{Information sources}

The following databases will be used to search for published, in progress and grey literature: MEDLINE (in process and 1947-date), EMBASE (1947-present), The Cochrane Central Register of Controlled Trials (CENTRAL), WHO International Clinical Trials Registry Platform (ICTRP) [26], ClinicalTrials.gov, The Global Index Medicus (GIM) [27] and Greenfile [28]. Furthermore, the use of manual searches of all reference lists in the included studies and previous systematic reviews related to the topics will ensure capture of all available literature. The systematic reviews will be identified whilst screening the search results for included studies and additionally searching Epistemonkios [29].

\section{Search strategy}

The search strategy, where the database platform allows, will include free-text terms and index terms that are contained within the following structure: "Population" AND ("Intervention" ("Household Air pollution" AND "LMICs")) (Appendix), with population being defined as pregnant women and children under 5 and interventions being any intervention 
that aims to reduce the level of household air pollution. There will be no restrictions in place for the date of publication, language of publication, type of publication (e.g. conference abstracts) or type of study design.

\section{Study records \\ Selection process}

Two reviewers (KEW, EDC) will independently conduct article selection using the eligibility criteria, within Mendeley, after removal of duplicates. Relevant articles will be determined initially by title and abstracts, followed by retrieval and full paper assessment for selection of papers as per the inclusion criteria, with reasons for exclusion noted at each stage (including the screening stage). Authors will be contacted for clarification if required. Any difference in selected articles between reviewers will be discussed using a third independent reviewer (SEB) to adjudicate any remaining disagreements. The selection process will be graphically illustrated using a PRISMA flow diagram [24].

\section{Data extraction}

Data will be independently extracted from included studies by two reviewers (KEW, EDC) using an adapted (to type of study design) Cochrane Public Health Group data extraction form [30], in a Microsoft Excel spreadsheet (Microsoft Cooperation). The data extraction form will include critically appraisal of paper quality within the assessment process. Extracted data will include, but not be limited to:

- Study characteristics

- Article title, author and year, geographical setting, study population characteristics (sex, age, residential setting), inclusion and exclusion criteria, funding sources

- Intervention details

- Type of interventions and comparators, length of intervention, baseline imbalances, issues with uptake and adoption

- Type of air pollution measurements, length of measurement, equipment used and results (if any)

- Health outcomes

- Health outcomes and definitions, method of measurement/classification scales, appropriateness of method, time points measured

Given the likely variability between studies included in the review, in terms of design, population, intervention, comparator, outcomes and data type, the data extraction process will be piloted and then modified if required. Any differences between reviewers in data extracted will be discussed and using a third independent reviewer (SEB) to adjudicate any remaining disagreements.

\section{Quality and bias assessment}

Risk of bias will be assessed using the Effective Public Health Practice Project (EPHPP) quality assessment tool for quantitative studies [31] by two reviewers independently (KEW, EDC), assigning low, medium and high risk of bias for each individual study. For trials where a parallel control group is used, it is accepted that random allocation and the blinding of participants and outcome assessor may not be always possible, due to the nature of the interventions and settings.

\section{Data synthesis}

A narrative synthesis will be undertaken for each population-intervention-outcome triad (as indicated in Fig. 1) stratified by study design. Data collected will be tabulated reporting study characteristics, intervention, HAP exposure measurements (if any) and outcome details. It is likely that data may be reported in a mixture of formats for the same outcome (e.g. continuous data mean, proportion meeting a fixed change, risks/relative risks, odds ratios). In addition, there will be a range of health outcomes reported, as well as a mixture of type of interventions,

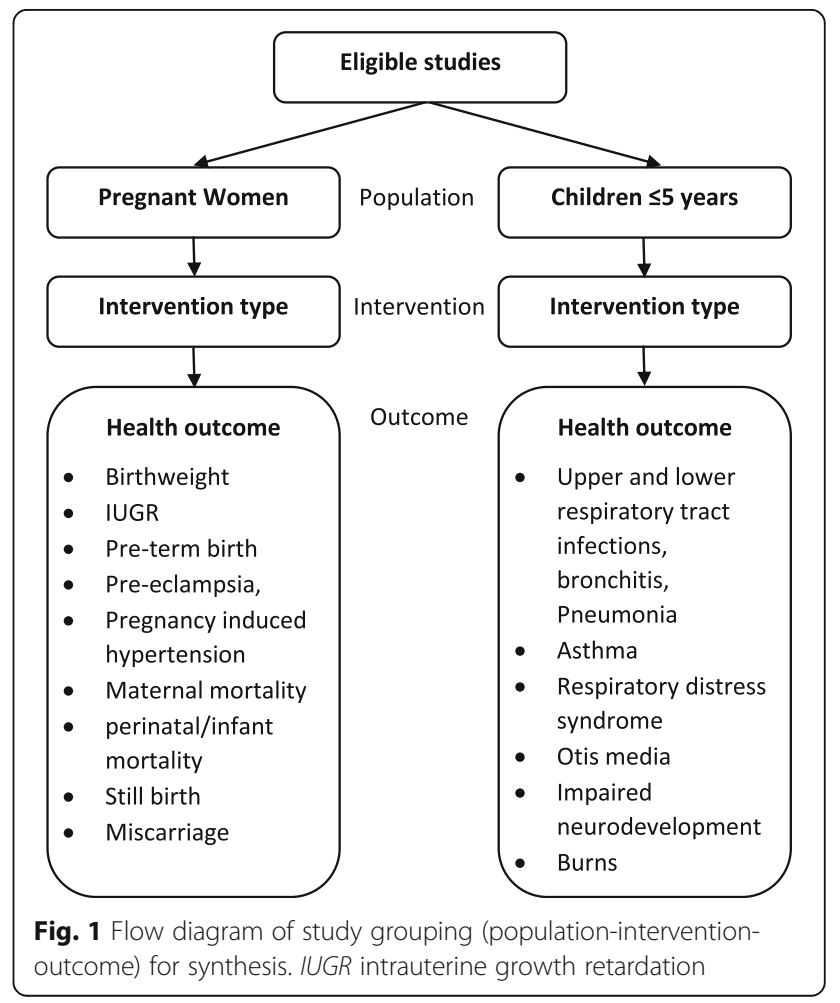


geographical regions and social contexts reported, which are likely to not be directly comparable.

Following on from the narrative analysis, metaanalysis will be considered within each triad, for each outcome measure, stratified by study design and the type of data available for the outcome. Clinical and methodological homogeneity within each triad will be used to determine the feasibility for meta-analysis where two or more studies in the same grouping report data in the same format at the same/similar time points. Any meta-analysis will be undertaken using a random effects model, due to an assumption that the studies represent a distribution of true effects. Determination of the level of between-study variation not attributable to chance will be calculated and displayed as an $I^{2}$ value with $95 \%$ confidence interval.

It is not anticipated that there will be more than a few studies in each meta-analysis, if even such an analysis is possible. The potential for additional sub-group analysis, sensitivity analysis or the assessment for the existence of small study effects using a funnel plot, will likely not exist. Risk of bias information will be used descriptively to contextualise the findings for each outcome whether a meta-analysis is undertaken or not. Recommendation for the improved conduct of studies in the field will be made.

\section{Discussion}

Alternatives to standard practices of household biomass fuel use for cooking, heating and lighting are required within LMICs to combat the morbidity and mortality presented by HAP, with implications for maternal and child health and sustainable economic development. National and local policymakers increasingly recognise the need for effective policy changes to mitigate the health burden associated with HAP exposure; however, there is a lack of evidence regarding affordable, effective and culturally acceptable interventions. This may restrict the progress of such changes, notably in countries which lack widespread access to mains electricity or gas for household cooking, heating and lighting, in addition to limits in transferability of effectiveness of interventions from one context to another. Harm mitigation approaches would bridge the gap before there is widespread affordable access to electricity or gas, but the efficacy of such an intervention requires evaluation. Therefore, this proposed review aims to report the contemporary scientific evidence concerning the effectiveness of HAP mitigation interventions to improve maternal and child health, thus identifying existing research gaps and informing future research and impact activities.

\section{Appendix}

\section{MEDLINE Search strategy}

\author{
Population \\ 1 (Child* adj3 (young or pre-school)).ti,ab. (72952) \\ 2 child, preschool/ or infant/ (1228593) \\ 3 (pregnan* or birth).ti,ab. (706117) \\ 4 (neonat* or infant or perinatal or newborn).ti,ab. (517748) \\ 5 exp Infant, Newborn/ (607223) \\ 6 foetus.ti,ab. (7397) \\ 7 Fetus/ (78154) \\ 8 fetus.ti,ab. (64247) \\ 9 (baby or babies).ti,ab. (69248) \\ 10 exp Pregnancy/ or exp Pregnant Women/ (894010) \\ 111 or 2 or 3 or 4 or 5 or 6 or 7 or 8 or 9 or $10(2660727)$
}

\section{Intervention}

12 ((clean* or modern) adj7 (energ* or fuel)).ti,ab. (2920)

13 (renewable* adj7 (energ* or fuel)).ti,ab. (4618)

14 (polic* adj7 (energ* or fuel)).ti,ab. (969)

15 (chang* $^{*}$ adj7 (energy* or fuel)).ti,ab. (23059)

16 exp Renewable Energy/ or exp Biofuels/ (31360)

17 ((solar or wind or hydro*) adj5 (energ* or power*)).ti,ab. (19581)

18 (behavio\$r adj9 (fuel or cook* or vent*)).ti,ab. (2091)

19 (transition adj7 (energ* or fuel)).ti,ab. (8426)

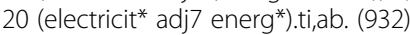

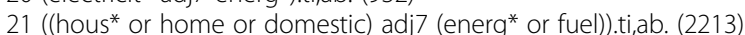

22 low polluting fuel*.ti,ab. (3)

23 (air adj7 ventilation).ti,ab. (3055)

24 (air pollution adj7 intervention).ti,ab. (73)

25 chimney.ti,ab. (1420)

26 "outdoor cook*".ti,ab. (9)

2712 or 13 or 14 or 15 or 16 or 17 or 18 or 19 or 20 or 21 or 22 or 23 or 24 or 25 or 26 (93856)

\section{Household air pollution}

28 ((household or indoor) adj3 air).ti,ab. (6933)

29 (HAP or IAP).ti,ab. (11561)

30 exp Air Pollution, Indoor/ (13569)

31 exp Particulate Matter/ (62709)

32 ("particulate matter" or "black carbon").ti,ab. (19909)

33 exp Carbon Monoxide/ (17931)

34 "carbon monoxide".ti,ab. (26660)

35 ((solid fuel or wood or charcoal or cook*) adj3 smok*).ti,ab. (1071)

36 (cookstove or stove).ti,ab. (1014)

37 Cooking/mt [Methods] (2210)

38 Household Articles/ (2254)

39 exp "Cooking and Eating Utensils"/ (1231)

4026 or 27 or 28 or 29 or 30 or 31 or 32 or 33 or 34 or 35 or 36 or 37 or 38 or 39 (222736)

\section{LMICs}

41 (LMIC or "lower adj3 middle income" or "developing countr" ).ti,ab. (60345) 42 exp Developing Countries/ (74829)

43 exp Africa, Western/ or exp Africa, Northern/ or South Africa/ or exp Africa, Eastern/ or exp Africa, Central/ or exp "Africa South of the Sahara"/ or exp Africa/ or exp Africa, Southern/ (266418)

44 Africa.tiab. (109048)

45 exp South America/ (161665)

$46 \exp$ Asia, Central/ or exp Asia, Northern/ or exp Asia/ or exp Asia, Western/ or exp Asia, Southeastern/ (835914)

47 south America.tiab. (14583)

48 Latin America.ti,ab. (13762)

49 Asia.ti,ab. (59583)

50 (Afghanistan or Albania or Algeria or Angola or "Antigua and Barbuda" or Argentina or Armenia or Azerbaijan or Bangladesh or Belarus or Belize or Benin or Bhutan or Bolivia or "Bosnia and Herzegovina" or Botswana or Brazil or Burkina Faso or Burundi or Cabo Verde or Cambodia or Cameroon or Central African Republic or Chad or China or Colombia or Comoros or Democratic 


\section{MEDLINE Search strategy (Continued)}

Republic of Congo or Congo or Cook Islands or Costa Rica or Cote d'Ivoire or Cuba or Djibouti or Dominica or Dominican Republic or Ecuador or Egypt or El Salvador or Equatorial Guinea or Eritrea or Ethiopia or Fiji or Gabon or Gambia or Georgia or Ghana or Grenada or Guatemala or Guinea or Guinea-Bissau or Guyana or Haiti or Honduras or India or Indonesia or Iran or Iraq or Jamaica or Jordan or Kazakhstan or Kenya or Kiribati or Democratic People's Republic of Korea or Kosovo or Kyrgyzstan or Lao People's Democratic Republic or Lebanon or Lesotho or Liberia or Libya or Former Yugoslav Republic of Macedonia or Madagascar or Malawi or Malaysia or Maldives or Mali or Marshall Islands or Mauritania or Mauritius or Mexico or Micronesia or Moldova or Mongolia or Montenegro or Montserrat or Morocco or Mozambique or Myanmar or Namibia or Nauru or Nepal or Nicaragua or Niger or Nigeria or Niue or Pakistan or Palau or Panama or Papua New Guinea or Paraguay or Peru or Philippines or Rwanda or Saint Helena or Samoa or "Sao Tome and Principe" or Senegal or Serbia or Sierra Leone or Solomon Islands or Somalia or South Africa or South Sudan or Sri Lanka or Saint Lucia or "Saint Vincent and the Grenadines" or Sudan or Suriname or Swaziland or Syrian Arab Republic or Tajikistan or Tanzania or Thailand or Timor-Leste or Togo or Tokelau or Tonga or Tunisia or Turkey or Turkmenistan or Tuvalu or Uganda or Ukraine or Uzbekistan or Vanuatu or Venezuela or Vietnam or "Wallis and Futuna" or "West Bank and Gaza Strip" or Yemen or Zambia or Zimbabwe).tiab. (1000461) 5141 or 42 or 43 or 44 or 45 or 46 or 47 or 48 or 49 or 50 (1807589)

\section{Grouped terms}

5211 and 27 (2227)

5340 and 51 (25902)

5411 and (27 or 53) (4306)

\section{Abbreviations}

ALRI: Acute lower respiratory infection; ARI: Acute respiratory infection; CENT RAL: The Cochrane Central Register of Controlled Trials; CO: Carbon monoxide; EPHPP: Effective Public Health Practice Project; GIM: Global Index Medicus; HAP: Household air pollution; ICS: Improved cookstove; ICTRP: International Clinical Trials Registry Platform; IUGR: Intrauterine growth retardation; LMICs: Low- and middle-income countries; LPG: Liquefied petroleum gas; RCT: Randomised control trial; PM: Particulate matter; PICOS: Population-Intervention-Comparator-Outcome-Study design; PRIS MA: Preferred Reporting Items for Systematic Reviews and Meta-Analyses; PRISMA-P: Preferred Reporting Items for Systematic Reviews and MetaAnalyses Protocol; PROSPERO: International Prospective Register of Systematic Reviews; WHO: World Health Organization

\section{Acknowledgements}

We are grateful to Sophie Bees for her review and comments on the methodology of the systematic review.

\section{Authors' contributions}

KEW and EDC initiated the aims and scope of the review under the supervision of SEB, GNT and SMH. Search strategy support was provided by AC. Methodological, support, advice and review were given by DJM. The manuscript was written by KEW and edited by EDC, DJM, GNT, SEB, FDP, SMH, RD, TO, BK, STM, TK, AS, WRA and DW. All authors have read and approved the manuscript for publication. GNT will be the guarantor for this review.

\section{Funding}

KW holds a University of Birmingham Global Challenges PhD scholarship, and the funder is independent of the systematic review.

\section{Availability of data and materials}

Not applicable, as no data was generated for this article

Ethics approval and consent to participate

Not applicable

\section{Consent for publication}

Not applicable

\section{Competing interests}

The authors have no competing interests to declare.

\section{Author details}

${ }^{1}$ Institute of Applied Heath Research, University of Birmingham, Birmingham, UK. ${ }^{2}$ Makerere University Lung Institute, College of Health Sciences, Mulago Hospital, Kampala, Uganda. ${ }^{3}$ University of Rwanda College of Science and Technology, Kigali, Rwanda. ${ }^{4}$ School of Geography, Earth and Environmental Sciences, University of Birmingham, Edgbaston, Birmingham, UK.

${ }^{5}$ International Development Department, University of Birmingham, Edgbaston, Birmingham, UK. ${ }^{6}$ Children's Hospital Los Angeles, University of Southern California, Los Angeles, USA.

Received: 16 August 2020 Accepted: 11 January 2021

Published online: 20 January 2021

\section{References}

1. Balakrishnan K, Parikh J, Sankar S, Padmavathi R, Srividya K, Venugopal V, et al. Daily average exposures to respirable particulate matter from combustion of biomass fuels in rural households of southern India. Environ Health Perspect. 2002;110:1069-75. https://doi.org/10.1289/ehp.021101069 PMID:12417476.

2. Pope D, Diaz E, Smith-Sivertsen T, Lie RT, Bakke P, Balmes JR, et al. Exposure to household air pollution from wood combustion and association with respiratory symptoms and lung function in nonsmoking women: results from the RESPIRE trial. Guatemala. Environ Health Perspect. 2015;123(4):28592. https://doi.org/10.1289/ehp.1408200.

3. Smith KR. Fuel combustion, air pollution exposure, and health: the situation in developing countries. Annu Rev Environ Resour. 1993;18:529-66.

4. Gakidou E, Afshin A, Abajobir AA, KH AK, Abbafati C, KM AK, et al. Global, regional, and national comparative risk assessment of 84 behavioural, environmental and occupational, and metabolic risks or clusters of risks, 1990-2016: a systematic analysis for the Global Burden of Disease Study 2016. Lancet. 2017:390(10100):1345-422. https://doi.org/10.1016/S01406736(17)32366-8 PMID:28919119.

5. Okello G, Devereux G, Semple S. Women and girls in resource poor countries experience much greater exposure to household air pollutants than men: results from Uganda and Ethiopia. Environ Int. 2018;119:429-37. https://doi.org/10.1016/j.envint.2018.07.002 PMID:30029097.

6. Dutta A, Brito K, Khramstova G, Mueller A, Chinthala S, Alexander D, et al. Household air pollution and angiogenic factors in pregnant Nigerian women: a randomized controlled ethanol cookstove intervention. Sci Total Environ. 2017;599:2175-81. https://doi.org/10.1016/j.scitotenv.2017.05.130.

7. Amegah AK, Quansah R, Jaakkola JJK. Household air pollution from solid fuel use and risk of adverse pregnancy outcomes: a systematic review and meta-analysis of the empirical evidence. PLoS One. 2014;9(12). https://doi. org/10.1371/journal.pone.0113920 PMID:25463771.

8. WHO (World Health Organization). Air pollution and child health: prescribing clean air. 2018. Available from: https://www.who.int/ceh/ publications/air-pollution-child-health/en/

9. Misra P, Srivastava R, Krishnan A, Sreenivaas V, Pandav CS. Indoor air pollution-related acute lower respiratory infections and low birthweight: a systematic review. J Trop Pediatr. 2012;58:457-66. https://doi.org/10.1093/ tropej/fms017 PMID:22555386.

10. Global Burden of Disease. GBD Compare |IHME Viz Hub. 2019. Available from: https:/vizhub.healthdata.org/gbd-compare/

11. World Health Organization (WHO). WHO Guidelines for indoor air quality: selected pollutants. Denmark; 2010. Available from: www.euro.who.int

12. Dohoo C, Guernsey JR, Critchley K, VanLeeuwen J. Pilot study on the impact of biogas as a fuel source on respiratory health of women on rural Kenyan smallholder dairy farms. J Environ Public Health. 2012:1-9. https://doi.org/10 1155/2012/636298 PMID:22969815.

13. Thomas E, Wickramasinghe K, Mendis S, Roberts N, Foster C. Improved stove interventions to reduce household air pollution in low and middle income countries: a descriptive systematic review. BMC Public Health. 2015;15:650. https://doi.org/10.1186/s12889-015-2024-7 PMID:26169364.

14. lessa L, De Vries YA, Swinkels CE, Smits M, Butijn CAA. What's cooking? Unverified assumptions, overlooking of local needs and pro-solution biases 
in the solar cooking literature. Energy Res Soc Sci. 2017;28:98-108. https:// doi.org/10.1016/j.erss.2017.04.007.

15. Bruce N, Pope D, Rehfuess E, Balakrishnan K, Adair-Rohani H, Dora C. WHO indoor air quality guidelines on household fuel combustion: strategy implications of new evidence on interventions and exposurerisk functions. Atmos Environ. 2015;106:451-7. https://doi.org/10.1016/j. atmosenv.2014.08.064.

16. Pope D, Bruce N, Dherani M, Jagoe K, Rehfuess E. Real-life effectiveness of 'improved' stoves and clean fuels in reducing PM 2.5 and CO: systematic review and meta-analysis. Environ Int. 2017;101:7-18. https://doi.org/10. 1016/..envint.2017.01.012 PMID:28285622.

17. Quansah R, Semple S, Ochieng CA, Juvekar S, Armah FA, Luginaah I, et al. Effectiveness of interventions to reduce household air pollution and/or improve health in homes using solid fuel in low-and-middle income countries: a systematic review and meta-analysis. Environ Int. 2017;103:7390. https://doi.org/10.1016/j.envint.2017.03.010 PMID:28341576.

18. Pennise D, Brant S, Mahu Agbeve S, Quaye W, Mengesha F, Tadele W, et al. Indoor air quality impacts of an improved wood stove in Ghana and an ethanol stove in Ethiopia. Energy Sustain Dev Indoor. 2003;13:71-6. https:// doi.org/10.1016/j.esd.2009.04.003.

19. Rehfuess E, Puzzolo E, Stanistreet D, Pope D, Bruce N. Enablers and barriers to large-scale uptake of improved solid fuel stoves: a systematic review. Env Heal Perspect. 2014;122(2):120-130.

20. Quinn AK, Bruce N, Puzzolo E, Dickinson K, Sturke R, Jack DW, et al. An analysis of efforts to scale up clean household energy for cooking around the world. Energy Sustain Dev. 2018;46:1-10. https://doi.org/10.1016/j.esd. 2018.06.011.

21. Thakur M, Nuyts PWAW, Boudewijns EA, Kim JF, Faber T, Babu GR. Impact of improved cookstoves on women's and child health in low and middle income countries: a systematic review and meta-analysis. Environ Expo. https://doi.org/10.1136/thoraxjnl-2017-210952 PMID:29925674.

22. Onakomaiya D, Gyamfi J, Iwelunmor J, Opeyemi J, Oluwasanmi M, ObiezuUmeh C, et al. Implementation of clean cookstove interventions and its effects on blood pressure in low-income and middle-income countries: systematic review. BMJ Open. 2019;9:e026517. https://doi.org/10.1136/ bmjopen-2018-026517.

23. PROSPERO International prospective register of systematic reviews. Available from: https://www.crd.york.ac.uk/prospero/. Accessed 16 Aug 2020.

24. Moher D, Liberati A, Tetzlaff J, Altman DG, Altman D, Antes G, et al. Preferred reporting items for systematic reviews and meta-analyses: the PRIS MA statement. PLoS Medicine. 2009;6:e1000097. https://doi.org/10.1371/ journal.pmed.1000097 PMID:19621072.

25. The World Bank Group. World Bank Country and Lending Groups. 2019. Available from: https://datahelpdesk.worldbank.org/knowledgebase/articles/ 906519

26. WHO (World Health Organization). International Clinical Trials Registry Platform (ICTRP): World Health Organization; 2020. Available from: http:// www.who.int/ictrp/en/

27. WHO (World Health Organization). Global Index Medicus. 2020; Available from: https://www.globalindexmedicus.net/.

28. EBSCO. GreenFILE. 2020. Available from: https://www.ebsco.com/products/ research-databases/greenfile

29. Epistemonikos. Epistemonikos: database of the best evidence-based health care. 2020. Available from: https://www.epistemonikos.org/en/

30. Cochrane Effective Practice and Organisation of Care (EPOC). Data collection form. EPOC resources for review authors. 2017. Available from: epoc. cochrane.org/resources/epoc-specific-resources-review-authors.

31. EPHPP (Effective public health practice Project). Quality assessment tool for quantitative studies. 1998. Available from: https://www.ephpp.ca/PDF/ Quality Assessment Tool_2010_2.pdf

\section{Publisher's Note}

Springer Nature remains neutral with regard to jurisdictional claims in published maps and institutional affiliations.

Ready to submit your research? Choose BMC and benefit from:

- fast, convenient online submission

- thorough peer review by experienced researchers in your field

- rapid publication on acceptance

- support for research data, including large and complex data types

- gold Open Access which fosters wider collaboration and increased citations

- maximum visibility for your research: over $100 \mathrm{M}$ website views per year

At BMC, research is always in progress.

Learn more biomedcentral.com/submissions 